\section{Efficient and Convenient Nonaqueous Workup Procedure for the Preparation of Arylboronic Esters}

\author{
Ken-Tsung Wong,* Yuh-Yih Chien, Yuan-Li Liao, \\ Chang-Chih Lin, Meng-Yen Chou, and Man-kit Leung \\ Department of Chemistry, National Taiwan University, \\ Taipe 106, Taiwan
}

kenwong@ccms.ntu.edu.tw

Received November 14, 2001

\begin{abstract}
An efficient one-pot synthetic protocol for the synthesis of arylboronic esters has been established. The concentrated addition mixture of trimethylborate with aryl Grignard reagents was treated with low molecular weight diols (ethylene glycol, 1,3-propandiol) and toluene, the corresponding arylboronic esters were isolated in a convenient way with high yields. The diols not only serve as water replacement for the workup step, but also as well as the reagent for the preparation of arylboronic esters.
\end{abstract}

Suzuki cross-coupling reactions of aryl halides and aryl triflates with arylboronic acids have been widely used for the construction of the aryl-aryl bond in modern organic synthesis. ${ }^{1}$ Much of the recent endeavors on the Suzuki coupling reaction have been concentrated on the metal ligand system. In particular, new catalyst systems ${ }^{2}$ that facilitate the cross-coupling of the less reactive aryl chlorides with boronic acids have been devel oped, greatly expanding the scope of the reaction. Some efforts have also been devoted to devel op new organoboron reagents. For instance, aryltrifluoroborate salts ${ }^{3}$ have been demonstrated to be as effective (in some cases better) at coupling with arenediazonium tetrafluoroborates, ${ }^{4}$ diaryliodonium salts, ${ }^{5}$ and aryl triflates ${ }^{6}$ in the presence of Pd catalyst. Despite that, the most popular arylboron derivatives used for Suzuki coupling reactions are arylboronic acids, which could be prepared by reacting a corresponding aryllithium or arylmagnesium complex with trialkyl borate, followed by an acidic aqueous workup. ${ }^{7}$ However, the aqueous workup procedure was sometimes troublesome and led to boronic acid only in moderate yield. In addition, the aqueous solution con6359 .

* To whom correspondence should be addressed. Fax: +886-2-2363-

(1) (a) Miyaura, N.; Suzuki, A. Chem. Rev. 1995, 95, 2457. (b) Suzuki, A. J . Organomet. Chem. 1999, 675, 147.

(2) (a) Littke, A.; Fu, G. C. Angew. Chem., Int. Ed. Engl. 1998, 37, 3387. (b) Old, D. W.; Wolfe, J . P.; Buchwald, S. L. J . Am. Chem. Soc. 1998, 120, 972. (c) Wolfe, J. P.; Buchwald, S. L. Angew. Chem., Int. Ed. Engl. 1999, 38, 2413. (d) Singer, R. A.; Buchwald, S. L. Tetrahedron Lett. 1999, 40, 1095. (e) Zhang, C.; Huang, J .; Trudell, M. L.; Nolan, S. P. J . Org. Chem. 1999, 64, 3804. (f) Littke, A. F.; Dai, C.; Fu, G. C. J. Am. Chem. Soc. 2000, 122, 4020. (g) Zapf, A.; Beller, M. Chem. Eur. J. 2000, 6, 1830.

(3) Vedejs, E.; Chapman, R. W.; Fields, S. C.; Lin, S.; Schrimpf, M. R. J . Org. Chem. 1995, 60, 3020.

(4) (a) Darses, S.; Genêt, J .-P.; Brayer, J .-L.; Demoute, J .-P. Tetrahedron Lett. 1997, 38, 4393. (b) Darses, S.; Michaud, G.; Genêt, J .-P. Eur. J . Org. Chem. 1999, 1875.

(5) Xia, M.; Chen, Z.-C. Synth. Commun. 1999, 29, 2457.

(6) Molander, G. A.; I to, T. Org. Lett. 2001, 3, 393.

(7) Diorazio, L. J .; Widdowson, D. A.; Clough, J. M. Tetrahedron 1992, 48, 8073 .

\section{Scheme 1. One-Pot Nonaqueous Workup} Synthesis of Arylboronic Esters

\begin{tabular}{|c|c|c|c|c|}
\hline & $\mathrm{B}(\mathrm{OMe})_{3}$ & concentration & $\mathrm{HO}\left(\mathrm{CH}_{2}\right)_{2} \mathrm{OH}$ & \\
\hline
\end{tabular}

taminated with boronic residue is not disposable, making this procedure not environmentally benign for large-scale preparation of the arylboronic acids. On the other hand, arylboronic esters ${ }^{8}$ have been used as a substitution of arylboronic acids to efficiently couple with aryl halides or aryl triflates in the presence of Pd-catalyst. Traditionally, arylboronic esters were prepared by a two-step synthesis: ${ }^{9}$ from aryl halides to aryl boronic acids, followed by treating the arylboronic acids with corresponding alcohol or diol in organic solvent. Alternatively, arylboronic esters can be obtained by $\mathrm{PdCl}_{2}(\mathrm{dppf})$-catalyzed boration of aryl halides or aryl triflates with dialkoxyborane. ${ }^{10}$ In this case, a small amount of the dehalogenated product is obtained as the side product. Pdcatalyzed borations of aryl halides or aryl triflates with tetraalkoxydi boron ${ }^{11}$ are more successful, leading to the formation of arylboronic esters with high yield. A broad range of functional groups are tolerable in these $\mathrm{Pd}$ catalyzed borations makes these methodologies attractive. In addition, Rh-catalyzed aromatic $\mathrm{C}-\mathrm{H}$ bond activation for the regioselective boration with pinacolborane in an inert solvent also provides the corresponding boronic ester efficiently. ${ }^{12}$ However, the dialkoxyborane is hygroscopic and the tetraalkoxydiboron is expensive, thus limiting these catalytic methods to be suitable only for small-scale preparation. Therefore, an environment friendly method for large-scale preparation of arylboronic esters with high efficiency is highly desired. In this paper, we report a convenient one-pot process for the preparation of arylboronic esters.

Arylboronic esters can be prepared by a one-pot procedure as shown in Scheme 1. Aryl bromide and aryl iodide are widely available from the major chemical suppliers at reasonable prices. The transformation from aryl halides to their Grignard reagents with magnesium metal in ethereal solvents (e.g., THF, diethyl ether) has been well documented. At $-78{ }^{\circ} \mathrm{C}$, the aryl Grignard reagents formed were added into the THF solution containing an excess amount (2 equiv) of trimethylborate, i.e., $\mathrm{B}(\mathrm{OMe})_{3}$. The reaction mixture was allowed to return to room temperature by removing the cooling bath, leading to the formation of aryl dimethoxyboronate. Instead of quenching the reaction mixture with acidic aqueous solution, the solvent and the excess amount of $\mathrm{B}(\mathrm{OMe})_{3}$ were removed by a rotary evaporator at ca. 20

(8) (a) Giroux, A.; Han, Y.; Prasit, P. Tetrahedron Lett. 1997, 38. 3841. (b) Wang, S.; Oldham, W. J .; Hudack, R. A.; Bazan, G. C. J . Am. Chem. Soc. 2000, 122, 5695. (c) Kristensen, J .; Lysen, M. Vedso, P. Begtrup, M. Org. Lett. 2001, 3, 1435.

(9) (a) Longstaff, C.; Rose, M. E. Org. Mass Spectrom. 1982, 17, 508. (b) Diorazio. L. J .; Widdowson, D. A.; Clough, J . M. Tetrahedron 1992, 48, 8073.

(10) (a) Murata, M.; Oyama, T.; Watanabe, S.; Masuda, Y. J . Org. Chem. 2000, 65, 164. (b) Baudoin, O.; Guénard, D.; Guéritte, F. J . Org. Chem. 2000, 65, 9268

(11) (a) I shiyama, T.; Murata, M.; Miyaura, N. J . Org. Chem. 1995, 60, 7508. (b) I shiyama, T.; I toh, Y.; Kitano, T.; Miyaura, N. Tetrahedron Lett. 1997, 38. 3447.

(12) Tse, M. K.; Cho, J .-Y.; Smith, III, M. R. Org. Lett. 2001, 3, 2831. 
Table 1. I solated Yield of One-Pot Nonaqueous Synthesis of Arylboronic Esters

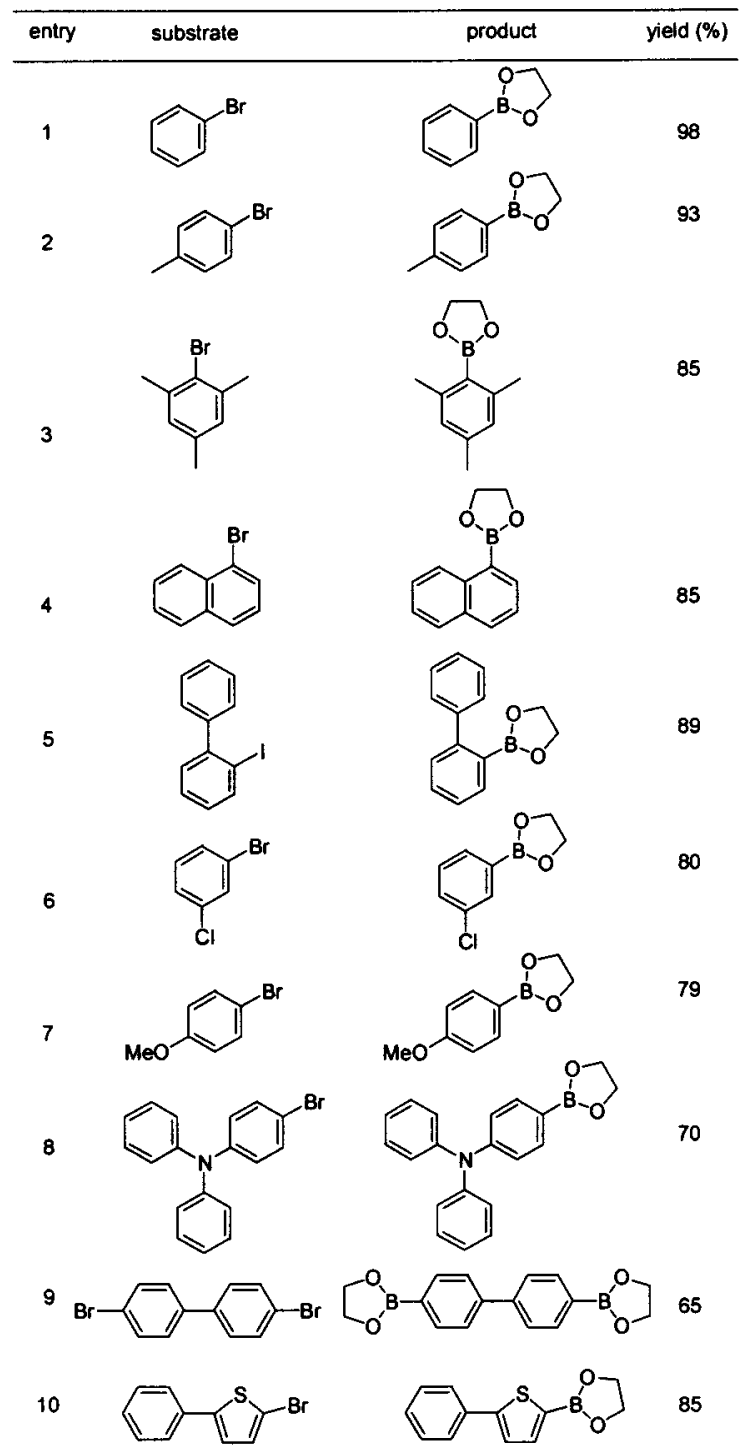

Torr to give a yellow white solid. The solid was found to be completely soluble in ethylene glycol, giving a clear solution. The resulting ethylene glycol solution was added with toluene and heated to reflux overnight. The toluene solution was separated and concentrated to afford the corresponding arylboronic ester as a single product.

According to this procedure, a $29 \mathrm{~g}$ of phenyl ethylene glycol boronic ester, ${ }^{13}$ which corresponded to a $98 \%$ yield, was isolated by reduced pressure distillation. To test the scope and limitation of this protocol, a variety of aryl halides with different structural feature were screened. The isolated yiel ds of arylboronic esters were usually good to excellent (Table 1). 4-Methylphenyl boronic ester was isolated in $93 \%$ yield as a colorless oil (entry 2 ). This method was also effective for the synthesis of bulky aryl boronic esters. For example, 2,4,6-trimethyl phenylboronic ester (mesitylene boronic ester) was isolated with a yield of $85 \%$ (entry 3), while steric hindered 1-naphthylboronic ester was isolated in $85 \%$ yield (entry 4). It is worthy to note that the 2-biphenylboronic acid ${ }^{14}$ was isolated only in $51 \%$ yield by a conventional hydrolytic

(13) Kaminski, J . J .; Lyle, R. E. Org. Mass Spectrom. 1978, 13, 425

(14) Aranyos, A.; Old, D. W.; Kiyomori, A.; Wolfe, J. P.; Sadighi, J . P.; Buchwald, S. L. J . Am. Chem. Soc. 1999, 121, 4369.

\section{Scheme 2. One-Pot Synthesis of Arylboronic Esters with Various Diols}

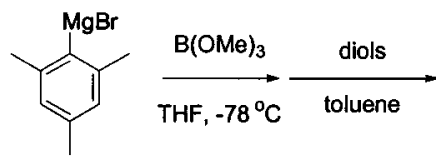

mesityl boronic ester

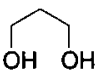<smiles>OB1CCCCO1</smiles>
$\mathrm{OH} \mathrm{OH}$ $80 \%$

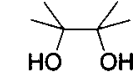<smiles>Cc1cc(C)c(B2OC(C)(C)C(C)(C)O2)c(C)c1</smiles>

$\mathrm{EtO}_{2}$<smiles>CCO[C@H]1OB(c2c(C)cc(C)cc2C)O[C@@H]1CC</smiles>

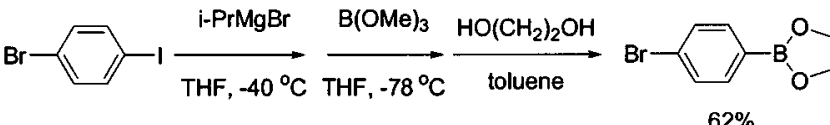

workup procedure. By taking advantage of the one-pot, nonaqueous workup procedure, 2-biphenyl boronic esters were isolated in $89 \%$ yield as a colorless oil (entry 5 ). The chemoselective Grignard reagent formation at the bromidesubstituted aryl carbon in the presence of a m-chloro group provides a pathway to synthesize the bifunctional reagent. Accordingly, 3-chlorophenylboronic ester was isolated in $80 \%$ yield by using our new procedure (entry 6). The phenyl groups with strong electron-donating substituents were also tested for the efficiency of this new procedure, phenyl groups with a para-substituted diphenylamino group or a methoxy group (entries 7 and 8) give the corresponding boronic esters in lower yields, 79\% for $4-\mathrm{OCH}_{3}$-phenyl and $70 \%$ for $4-\mathrm{Ph}_{2} \mathrm{~N}$-phenyl. Aryldiboronic esters are important building blocks for the synthesis of $\pi$-conjugated oligomers and polymers for the optoelectronic uses. ${ }^{15}$ According to our new method, the $4,4^{\prime}$-biphenyldiboronic ester was isolated in $65 \%$ yield (entry 9). Heteroaryl halides were also suitable for this new method; 2-(5-phenylthienyl) boronic ester was isolated in $85 \%$ yield (entry 10 ).

In addition to using ethylene glycol for this one-pot protocol, 1,3-propanediol, pinacol, and diethyl L-tartrate are al so applicable for this new procedure to provide the corresponding boronic ester (Scheme 2). The treatment of mesitylenemagnesium bromide with trimethyl borate followed by reaction with 1,3-propanediol gave the boronic ester in $80 \%$ yield. 1,3-Propanediol that is insoluble in toluene acts as conveniently as ethylene glycol, providing a well-separated two-phase solution. Thus, the corresponding mesityleneboronic ester can be easily isolated from the toluene solution with comparable yield. How-

(15) (a) Beley, M.; Chodorowski, S.; Collin, J.-P.; Sauvage, J .-P. Tetrahedron Lett. 1993, 34, 2933. (b) Yu, W.-L.; Pei, J .; Huang, W.; Heeger, A. J . Chem. Commun. 2000. 681. (c) Yu, W.-L.; Huang, W. Heeger, A. J . Adv. Mater. 2000, 12, 828. 
ever, when the pinacol and diethyl L-tartrate were used instead of ethylene glycol, the corresponding boronic esters were isolated in $81 \%$ and $82 \%$ yield, respectively. In these two cases, there are no well-separated two-layer solutions after refluxing overnight due to the partial solubility of pinacol and diethyl L-tartrate in toluene. The isolation of the product was accomplished by directly distilling the resulting mixture under reduced pressure. To widen the scope of this new procedure, the method used to generate the Grignard reagents is not only limited to directly transform from the aryl halides with magnesium metal, but can also be generated by Knochel's exchanging protocol. ${ }^{16}$ Accordingly, when 4-iodobromobenzene in THF was treated with ${ }^{i} \operatorname{PrMgBr}$ (2 equiv) at -40 ${ }^{\circ} \mathrm{C}$ for $3 \mathrm{~h}$, the resulting solution was further reacted with $\mathrm{B}(\mathrm{OMe})_{3}$ at $-78{ }^{\circ} \mathrm{C}$, followed by the new nonaqueous workup procedure giving 4-bromophenylboronic ester in $62 \%$ (Scheme 2). The varieties of the methods to generate the Grignard reagents and the different nature of the diols suitable for subsequently workup procedure widely expand the scope of this new procedure.

In summary, we have successfully established an efficient and convenient nonaqueous procedure for the large-scale preparation of arylboronic esters. In one pot, aryl halides that can be transformed into their corresponding Grignard reagents can easily be used for the preparation of aryl boronic ester. The lowest molecular weight diol, ethylene glycol, not only serves as a replacement of water for the workup step but also acts as the reagent for the synthesis of the corresponding boronic esters in good to excellent yields. The boronic residue was dissolved in ethylene glycol, leading to no boron-contaminated aqueous waste. Instead of using ethylene glycol, other diols such as 1,3-propanediol, pinacol, and diethyl L-tartrate are also effective for this new procedure.

\section{Experimental Section}

General Experimental Procedure. All reactions were performed under argon and were magnetically stirred. Solvents were distilled from an appropriate drying agent prior to use: $\mathrm{THF}$ and $\mathrm{Et}_{2} \mathrm{O}$ from sodium and benzophenone, toluene from sodium. Commercially available reagents were used without further purification unless otherwise stated. Melting points were measured on a Fargo MP-1D and are uncorrected. ${ }^{1} \mathrm{H}$ NMR and ${ }^{13} \mathrm{C}$ NMR were recorded using a Bruker (AC-400) or Varian (400 Unity Plus) spectrometer at 400 and $100 \mathrm{MHz}$, respectively. Lowand high-resolution mass spectra were recorded using a J EOL SX-102A spectrometer in EI mode. Microanalyses were carried out on a Perkin-EImer 240 analyzer.

Nonaqueous Preparation of Arylboronic Esters. General Procedure. Arylmagnesium halide (1.0 M ) prepared from aryl halide (1.0 equiv) and magnesium turnings (1.1 equiv) in THF was added dropwise into a solution of trimethyl borate (2.0 equiv) in THF at $-78^{\circ} \mathrm{C}$. The reaction mixture was warmed to room temperature and concentrated to dryness by rotary evaporation distillation (20 Torr). The resulting solid was added ethylene glycol and toluene. The mixture was refluxed overnight, and the toluene layer was separated and concentrated in vacuo to give the corresponding boronic ester.

Representative Procedure for the Nonaqueous Preparation of Arylboronic Esters: 2-Phenyl[1,3,2]dioxaborolane. ${ }^{17}$ Phenylmagnesium bromide prepared from bromobenzene $(21.0 \mathrm{~mL}, 200 \mathrm{mmol})$ and magnesium turning $(4.86 \mathrm{~g}, 200 \mathrm{mmol})$ in THF $(200 \mathrm{~mL})$ was added dropwise into a solution of trimethyl borate $(45.7 \mathrm{~mL}, 400 \mathrm{mmol})$ in THF $(200 \mathrm{~mL})$ at $-78^{\circ} \mathrm{C}$. The reaction mixture was warmed to room temperature and concen-

(16) Boymond, L.; Rottlander, M.; Gahiez, G.; Knochel, P. Angew. Chem., Int. Ed. Engl. 1998, 37, 1701.

(17) Kaminski, J .J .; Lyle, R. E. Org. Mass Spectrom. 1978, 13, 425. trated to dryness by rotary evaporator distillation (20 Torr). To the resulting solid were added ethylene glycol $(30 \mathrm{~mL})$ and toluene $(100 \mathrm{~mL})$. The mixture was refluxed overnight, and the toluene layer was separated and concentrated by simple distillation under nitrogen. 2-Phenyl[1,3,2]dioxaborolane (29 g, 98\%) was isolated as a colorless oil by distillation under reduced pressure: bp $53-54^{\circ} \mathrm{C}\left(0.4\right.$ Torr) (lit. ${ }^{17}$ bp $\left.57-59{ }^{\circ} \mathrm{C}\right) ;{ }^{1} \mathrm{H}$ NMR $\left(\mathrm{CDCl}_{3}, 400 \mathrm{MHz}\right) 4.37(\mathrm{~s}, 4 \mathrm{H}), 7.37-7.42(\mathrm{~m}, 2 \mathrm{H}), 7.47-7.49$ $(\mathrm{m}, 1 \mathrm{H}), 7.83(\mathrm{~d}, \mathrm{~J}=8.0 \mathrm{~Hz}, 2 \mathrm{H}) ;{ }^{13} \mathrm{C} \mathrm{NMR}\left(\mathrm{CDCl}_{3}, 400 \mathrm{~Hz}\right)$ $66.0,121.8,131.4,134.8$.

2-p-Tolyl[1,3,2]dioxaborolane. ${ }^{18}$ 4-Bromotoluene (3.42 g, 20 $\mathrm{mmol}), \mathrm{Mg}(0.53 \mathrm{~g}, 22 \mathrm{mmol})$, trimethyl borate $(4.16 \mathrm{~g}, 40 \mathrm{mmol})$, THF $(20 \mathrm{~mL})$, and ethylene glycol $(10 \mathrm{~mL})$, isolated as a white soild (3.01 g, 93\%): $\mathrm{mp} 59-60^{\circ} \mathrm{C}$ (lit. $\left.{ }^{17} \mathrm{mp} 60-61^{\circ} \mathrm{C}\right) ;{ }^{1} \mathrm{H} N M R$ $\left(\mathrm{CDCl}_{3}, 400 \mathrm{MHz}\right) \delta 2.39(\mathrm{~s}, 3 \mathrm{H}), 4.38(\mathrm{~s}, 4 \mathrm{H}), 7.21(\mathrm{~d}, \mathrm{~J}=7.68$ $\mathrm{Hz}, 2 \mathrm{H}), 7.72(\mathrm{~d}, \mathrm{~J}=7.68 \mathrm{~Hz}, 1 \mathrm{H}) ;{ }^{13} \mathrm{C} \mathrm{NMR}\left(\mathrm{CDCl}_{3}, 100 \mathrm{MHz}\right)$ $\delta$ 21.7, 65.9, 128.7, 134.9, 141.7; MS (EI, m/z) 162 (70); HRMS (EI) $\mathrm{m} / \mathrm{z}$ calcd for $\mathrm{C}_{9} \mathrm{H}_{11} \mathrm{BO}_{2} 162.0859$, found 162.0852 .

2-(2,4,6-Trimethylphenyl)[1,3,2] dioxaborolane. 1-Bromo2,4,6-trimethylbenzene $(1.0 \mathrm{~g}, 5.2 \mathrm{mmol}), \mathrm{Mg}(0.25 \mathrm{~g}, 10.4$ $\mathrm{mmol})$, trimethyl borate $(1.08 \mathrm{~g}, 10.4 \mathrm{mmol})$, THF $(10 \mathrm{~mL})$, ethylene glycol $(5 \mathrm{~mL})$, isolated as white sol id $(0.84 \mathrm{~g}, 85 \%): \mathrm{mp}$ 108-109 ${ }^{\circ} \mathrm{C} ;{ }^{1} \mathrm{H} \mathrm{NMR}\left(400 \mathrm{MHz} \mathrm{CDCl}_{3}\right) \delta 2.26(\mathrm{~s}, 3 \mathrm{H}), 2.37(\mathrm{~s}$, $6 \mathrm{H}), 4.36(\mathrm{~s}, 4 \mathrm{H}), 6.81(\mathrm{~s}, 2 \mathrm{H}) ;{ }^{13} \mathrm{C} \mathrm{NMR}\left(400 \mathrm{MHz} \mathrm{CDCl}_{3}\right)$ $\delta 21.20,22.49,65.56,127.62,139.35,142.87$; HRMS (FAB) calcd for $\mathrm{C}_{11} \mathrm{H}_{15} \mathrm{BO}_{2} 190.1163$, found 190.1165. Anal. Calcd for $\mathrm{C}_{11} \mathrm{H}_{15^{-}}$ $\mathrm{BO}_{2}: \mathrm{C}, 69.52 ; \mathrm{H}, 7.96$. Found: $\mathrm{C}, 69.44 ; \mathrm{H}, 8.20$.

2-(1-Naphthyl)[1,3,2]dioxaborolane. ${ }^{19}$ 1-Bromonaphthalene $(2.07 \mathrm{~g}, 10.0 \mathrm{mmol}), \mathrm{Mg}(0.27 \mathrm{~g}, 11.0 \mathrm{mmol})$, trimethyl borate $(2.08 \mathrm{~g}, 20.0 \mathrm{mmol}), \mathrm{THF}(10 \mathrm{~mL})$, ethylene glycol $(5 \mathrm{~mL})$, isolated as a white solid (1.68 g, 85\%): $\mathrm{mp} 57-58{ }^{\circ} \mathrm{C}$ (lit. ${ }^{19} \mathrm{mp}$ $\left.146-149^{\circ} \mathrm{C}\right) ;{ }^{1} \mathrm{H}$ NMR $\left(\mathrm{CDCl}_{3}, 400 \mathrm{MHz}\right) \delta 4.49(\mathrm{~s}, 4 \mathrm{H}), 7.48-$ $7.55(\mathrm{~m}, 3 \mathrm{H}), 7.86(\mathrm{~d}, \mathrm{~J}=8.8 \mathrm{~Hz}, 1 \mathrm{H}), 7.97(\mathrm{~d}, \mathrm{~J}=8.2 \mathrm{~Hz}, 1 \mathrm{H})$, $8.13(\mathrm{~d}, \mathrm{~J}=5.8 \mathrm{~Hz}, 1 \mathrm{H}), 8.76(\mathrm{~d}, \mathrm{~J}=8.9 \mathrm{~Hz}, 1 \mathrm{H}) ;{ }^{13} \mathrm{C} \mathrm{NMR}$ $\left(\mathrm{CDCl}_{3}, 100 \mathrm{MHz}\right) \delta 65.9,125.0,125.6,126.5,128.3,128.4,132.0$, 133.2, 136.1, 136.9; MS (EI, m/z) 198 (100); HRMS (EI) calcd for $\mathrm{C}_{12} \mathrm{H}_{11} \mathrm{BO}_{2}$ 198.0852, found 198.0865 .

2-(2-Biphenyl)[1,3,2]dioxaborolane. 2-Bromobi phenyl (2.79 $\mathrm{g}, 10.0 \mathrm{mmol}), \mathrm{Mg}(0.26 \mathrm{~g}, 11.0 \mathrm{mmol})$, trimethyl borate $(2.08 \mathrm{~g}$, $20.0 \mathrm{mmol})$, diethyl ether $(10 \mathrm{~mL})$, ethylene glycol $(5 \mathrm{~mL})$, isolated as a colorless oil $(2.01 \mathrm{~g}, 89 \%)$ : ${ }^{1} \mathrm{H} \mathrm{NMR}\left(\mathrm{CDCl}_{3}, 400\right.$ $\mathrm{MHz}) \delta 4.22(\mathrm{~s}, 4 \mathrm{H}), 7.35-7.42(\mathrm{~m}, 7 \mathrm{H}), 7.50(\mathrm{td}, \mathrm{J}=7.43,1.4$ $\mathrm{Hz}, 3 \mathrm{H}), 7.83(\mathrm{dd}, J=7.37,1.0 \mathrm{~Hz}, 1 \mathrm{H}) ;{ }^{13} \mathrm{C}$ NMR (acetone- $\mathrm{d}_{6}$, $100 \mathrm{MHz}) \delta 66.0,126.9,127.2,127.5,127.9,128.4,129.0,219.4$, 129.5, 131.0, 135.2, 140.7, 147.6; MS (EI , m/z) 224 (100); HRMS (EI) calcd for $\mathrm{C}_{9} \mathrm{H}_{11} \mathrm{BO}_{3} 178.0801$, found 178.0809.

2-(3-Chlorophenyl)[1,3,2]dioxaborolane. 3-Chlorobromobenzene $(3.83 \mathrm{~g}, 20.0 \mathrm{mmol}), \mathrm{Mg}(0.53 \mathrm{~g}, 22.0 \mathrm{mmol})$, trimethyl borate $(4.16 \mathrm{~g}, 40.0 \mathrm{mmol})$, THF $(20 \mathrm{~mL})$, ethylene glycol (10 $\mathrm{mL})$, isolated as a colorless oil $(2.92 \mathrm{~g}, 80 \%)$ : bp $68-70{ }^{\circ} \mathrm{C}(0.5$ Torr); ${ }^{1} \mathrm{H}$ NMR $\left(\mathrm{CDCl}_{3}, 400 \mathrm{MHz}\right) \delta 4.39(\mathrm{~s}, 4 \mathrm{H}), 7.31(\mathrm{t}, \mathrm{J}=7.7$ $\mathrm{Hz} 1 \mathrm{H}), 7.44(\mathrm{~d}, \mathrm{~J}=8.0 \mathrm{~Hz}, 1 \mathrm{H}), 7.67(\mathrm{~d}, \mathrm{~J}=7.3 \mathrm{~Hz}, 1 \mathrm{H}), 7.78$ $(\mathrm{s}, 1 \mathrm{H}) ;{ }^{13} \mathrm{C} \mathrm{NMR}\left(\mathrm{CDCl}_{3}, 100 \mathrm{MHz}\right) \delta 66.2,129.3,131.5,132.7$, 134.1, 134.7; MS (EI, m/z) 182 (43), 184 (14); HRMS (EI) calcd for $\mathrm{C}_{8} \mathrm{H}_{8} \mathrm{~B}^{35} \mathrm{ClO}_{2}$ 182.0306, found 182.0310; calcd for $\mathrm{C}_{8} \mathrm{H}_{8} \mathrm{~B}^{37}$. $\mathrm{ClO}_{2}$ 184.0276, found 184.0268 .

2-(4-Methoxylphenyl)[1,3,2]dioxaborolane.20 4-Bromoanisole $(3.74 \mathrm{~g}, 20 \mathrm{mmol}), \mathrm{Mg}(0.53 \mathrm{~g}, 22 \mathrm{mmol})$, trimethyl borate $(4.16 \mathrm{~g}, 40 \mathrm{mmol}), \operatorname{THF}(20 \mathrm{~mL})$, ethylene glycol $(10 \mathrm{~mL})$, isolated as a light yellow solid $(2.80 \mathrm{~g}, 79 \%)$ : $\mathrm{mp} 36-37{ }^{\circ} \mathrm{C}$ (lit. ${ }^{17} \mathrm{mp}$ $\left.50-52^{\circ} \mathrm{C}\right) ;{ }^{1} \mathrm{H}$ NMR $\left(\mathrm{CDCl}_{3}, 400 \mathrm{MHz}\right) \delta 3.84(\mathrm{~s}, 3 \mathrm{H}), 4.36(\mathrm{~s}$, $4 \mathrm{H}), 6.92(\mathrm{~d}, \mathrm{~J}=8.3 \mathrm{~Hz}, 3 \mathrm{H}), 7.77(\mathrm{~d}, \mathrm{~J}=8.3 \mathrm{~Hz}, 1 \mathrm{H}) ;{ }^{13} \mathrm{C} \mathrm{NMR}$ $\left(\mathrm{CDCl}_{3}, 100 \mathrm{MHz}\right) \delta 55.1,65.9,113.5,136.6,162.3 ; \mathrm{MS}(\mathrm{EI}, \mathrm{m} / \mathrm{z})$ 178 (100); HRMS (EI) calcd for $\mathrm{C}_{9} \mathrm{H}_{11} \mathrm{BO}_{3}$ 178.0801, found 178.0809.

2-(4-Diphenylaminophenyl)[1,3,2]dioxaborolane. (4-Bromophenyl)diphenylamine ${ }^{21}(1.0 \mathrm{~g}, 3.1 \mathrm{mmol}), \mathrm{Mg}(0.15 \mathrm{~g}, 6.2$ $\mathrm{mmol})$, trimethyl borate $(0.65 \mathrm{~g}, 6.2 \mathrm{mmol})$, THF $(10 \mathrm{~mL})$, ethylene glycol $(1.6 \mathrm{~mL})$, isolated as a waxy solid $(0.68 \mathrm{~g}, 70 \%)$ : ${ }^{1} \mathrm{H} \mathrm{NMR}\left(\mathrm{CDCl}_{3}, 400 \mathrm{MHz}\right) \delta 4.36(\mathrm{~s}, 4 \mathrm{H}), 7.15-7.03(\mathrm{~m}, 8 \mathrm{H})$, 7.30-7.26 (m, 4H), $7.67(\mathrm{~d}, \mathrm{~J}=8.6 \mathrm{~Hz}, 2 \mathrm{H}) ;{ }^{13} \mathrm{C} \mathrm{NMR}\left(\mathrm{CDCl}_{3}\right.$,

(18) Longstaff, C.; Rose, M. E. Org. Mass Spectrom. 1982, 17, 508

(19) Ingham, R. K.; Huntsman, W. D. Chem. Abstr. 1963, 58, 4587e.

(20) Wallow, T. I.; Novak, B. M. J. Org. Chem. 1994, 59, 9; 17.

(21) Creson, S. C.; Wheeler, J .; Nelson, R. F. J . Org. Chem. 1972 $37,4440$. 
$100 \mathrm{MHz}) \delta 65.96,121.43,123.56,125.22,129.35,135.93,147.29$; MS (EI, m/z) 315 (100); HRMS (FAB) calcd for $\mathrm{C}_{20} \mathrm{H}_{18} \mathrm{BNO}_{2}$ 315.1431, found 315.1427.

Biphenyl-4,4'-ethylene Glycol Diboronic Ester.22 4,4'Dibromobiphenyl (3.12 g, $10.0 \mathrm{mmol}), \mathrm{Mg}(0.53 \mathrm{~g}, 22.0 \mathrm{mmol})$, trimethyl borate $(4.16 \mathrm{~g}, 40.0 \mathrm{mmol})$, THF $(20 \mathrm{~mL})$, ethylene glycol $(5 \mathrm{~mL})$, isolated as a pale yellow solid $(1.91 \mathrm{~g}, 65 \%): \mathrm{mp}$ 288-289 ${ }^{\circ} \mathrm{C} ;{ }^{1} \mathrm{H} \mathrm{NMR}\left(\mathrm{CDCl}_{3}, 400 \mathrm{MHz}\right) \delta 4.41(\mathrm{~s}, 8 \mathrm{H}), 7.66(\mathrm{~d}$ $\mathrm{J}=4.9 \mathrm{~Hz}, 4 \mathrm{H}), 7.90(\mathrm{~d}, \mathrm{~J}=4.9 \mathrm{~Hz}, 4 \mathrm{H}) ;{ }^{13} \mathrm{C} \mathrm{NMR}\left(\mathrm{CDCl}_{3}, 100\right.$ MHz) $\delta 66.0,126.6,135.3,143.7$; MS (EI, m/z) 294(100); HRMS (EI) calcd for $\mathrm{C}_{16} \mathrm{H}_{16} \mathrm{~B}_{2} \mathrm{O}_{4}$ 294.1235, found 294.1240.

2-(5-Phenylthienyl)ethylene Glycol Boronic Ester. 2-Bromo-5-phenylthiophene ${ }^{23}(1.2 \mathrm{~g}, 5.0 \mathrm{mmol}), \mathrm{Mg}(0.132 \mathrm{~g}, 5.5$ $\mathrm{mmol})$, trimethyl borate $(1.04 \mathrm{~g}, 10.0 \mathrm{mmol})$, THF $(10 \mathrm{~mL})$, ethylene glycol ( $3 \mathrm{~mL})$, isolated as a white solid $(0.98 \mathrm{~g}, 85 \%)$ : $\mathrm{mp} 111-112^{\circ} \mathrm{C} ;{ }^{1} \mathrm{H} \mathrm{NMR}\left(\mathrm{CDCl}_{3}, 400 \mathrm{MHz}\right) \delta 4.40(\mathrm{~s}, 4 \mathrm{H}), 7.26-$ $7.33(\mathrm{~m}, 1 \mathrm{H}), 7.38-7.42(\mathrm{~m}, 3 \mathrm{H}), 7.66(\mathrm{~d}, \mathrm{~J}=3.6 \mathrm{~Hz}, 1 \mathrm{H}), 7.67$ $(\mathrm{d}, J=6.0 \mathrm{~Hz}, 2 \mathrm{H}) ;{ }^{13} \mathrm{C} \mathrm{NMR}\left(\mathrm{CDCl}_{3}, 100 \mathrm{MHz}\right) \delta 66.1,124.5$, 126.2, 128.0, 128.9, 134.0, 138.5, 151.7; MS (EI , m/z) 230 (100); HRMS (EI) calcd for $\mathrm{C}_{12} \mathrm{H}_{11} \mathrm{BO}_{2} \mathrm{~S} 230.0573$, found 230.0565 .

2-(2,4,6-Trimethylphenyl)[1,3,2]dioxaborinane. 1-Bromo2,4,6-trimethylbenzene $(3.98 \mathrm{~g}, 20.0 \mathrm{mmol}), \mathrm{Mg}(0.53 \mathrm{~g}, 22$ $\mathrm{mmol})$, trimethyl borate $(4.16 \mathrm{~g}, 40 \mathrm{mmol}), \operatorname{THF}(20 \mathrm{~mL}), 1,3-$ propanediol $(5 \mathrm{~mL})$, isolated as a white solid $(3.2 \mathrm{~g}, 80 \%)$ : $\mathrm{mp}$ 48-49 ${ }^{\circ} \mathrm{C} ;{ }^{1} \mathrm{H} \mathrm{NMR}\left(\mathrm{CDCl}_{3}, 400 \mathrm{MHz}\right) \delta 2.10$ (qn, J = $5.5 \mathrm{~Hz}$, $2 \mathrm{H}), 2.24(\mathrm{~s}, 3 \mathrm{H}), 2.33(\mathrm{~s}, 6 \mathrm{H}), 4.17(\mathrm{t}, \mathrm{J}=5.5 \mathrm{~Hz}, 4 \mathrm{H}), 6.77(\mathrm{~s}$, $2 \mathrm{H}) ;{ }^{13} \mathrm{C} \mathrm{NMR}\left(\mathrm{CDCl}_{3}, 100 \mathrm{MHz}\right) \delta 21.2,21.9,27.7,62.0,127.1$, 137.9, 140.3; MS (EI, m/z) 204 (100); HRMS (EI m/z) calcd for $\mathrm{C}_{12} \mathrm{H}_{17} \mathrm{BO}_{2}$ 204.1322, found 204.1322.

Mesitylpinacol Boronate Ester. 2-Bromo-2,4,6-trimethylbenzene $(3.98 \mathrm{~g}, 20.0 \mathrm{mmol}), \mathrm{Mg}(0.53 \mathrm{~g}, 22 \mathrm{mmol})$, trimethyl borate $(4.16 \mathrm{~g}, 40 \mathrm{mmol}), \operatorname{THF}(20 \mathrm{~mL})$, pinacol $(7.08 \mathrm{~g})$, isolated as a colorless oil $(4.0 \mathrm{~g}, 81 \%)$ : bp $125^{\circ} \mathrm{C}$ bulb-to-bulb (0.1 Torr); ${ }^{1} \mathrm{H} \mathrm{NMR}\left(\mathrm{CDCl}_{3}, 400 \mathrm{MHz}\right) \delta 1.38(\mathrm{~s}, 12 \mathrm{H}), 2.25(\mathrm{~s}, 3 \mathrm{H}), 2.38(\mathrm{~s}$

(22) Beley, M.; Chodorowski, S.; Collin, J .-P.; Sauvage, J .-P. Tetrahedron Lett. 1993, 34, 2933. 25 .

(23) Hotta, S.; Lee, S. A.; Tamaki, T. J . Heterocycl. Chem. 2000, 37,
$6 \mathrm{H}), 6.78(\mathrm{~s}, 2 \mathrm{H}) ;{ }^{13} \mathrm{C} \mathrm{NMR}\left(\mathrm{CDCl}_{3}, 100 \mathrm{MHz}\right) \delta 21.2,22.2,24.9$, 83.4, 127.4, 138.9, 142.1; MS (EI, m/z) 246 (100); HRMS (EI m/z) calcd for $\mathrm{C}_{15} \mathrm{H}_{23} \mathrm{BO}_{2}$ 246.1791, found 246.1794.

Mesityl Diethyl L-Tartrate Boronate Ester. 2-Bromo2,4,6-trimethylbenzene $(3.98 \mathrm{~g}, 20.0 \mathrm{mmol}), \mathrm{Mg}(0.53 \mathrm{~g}, 22$ $\mathrm{mmol})$, trimethyl borate $(4.16 \mathrm{~g}, 40 \mathrm{mmol}), \operatorname{THF}(20 \mathrm{~mL})$, diethyl L-tartrate $(10 \mathrm{~mL})$, isolated as a colorless oil $(5.47 \mathrm{~g}, 82 \%)$ : ${ }^{1} \mathrm{H}$ $\operatorname{NMR}\left(\mathrm{CDCl}_{3}\right) \delta 1.35(\mathrm{t}, \mathrm{J}=2.76 \mathrm{~Hz}, 6 \mathrm{H}), 2.29(\mathrm{~s}, 3 \mathrm{H}), 2.47(\mathrm{~s}$ $6 \mathrm{H}), 4.34(\mathrm{q}, \mathrm{J}=2.76 \mathrm{~Hz}, 4 \mathrm{H}), 5.04(\mathrm{~s}, 2 \mathrm{H}), 6.85(\mathrm{~s}, 2 \mathrm{H}) ;{ }^{13} \mathrm{C}$ $\mathrm{NMR}\left(\mathrm{CDCl}_{3}, 100 \mathrm{MHz}\right) \delta 14.1,21.3,22.4,62.2,127.8,169.7$, 140.2, 143.7; MS (EI, m/z) 334 (100); HRMS (EI m/z) calcd for $\mathrm{C}_{17} \mathrm{H}_{23} \mathrm{BO}_{6} 334.1588$, found 334.1580.

2-(4-Bromophenyl)[1,3,2]dioxaborolane. ${ }^{17}$ 2-Propylmagnesium bromide prepared from 2-bromopropane $(0.94 \mathrm{~mL}, 10$ $\mathrm{mmol})$ and $\mathrm{Mg}(0.243 \mathrm{~g}, 10 \mathrm{mmol})$ in THF $(10 \mathrm{~mL})$ was added dropwise into a solution of 4-iodobromobenzene (1.41 g, 5.0 $\mathrm{mmol})$ in THF $(10 \mathrm{~mL})$ at $-40{ }^{\circ} \mathrm{C}$. The mixture was stirred for $3 \mathrm{~h}$ and cooled to $-78^{\circ} \mathrm{C}$. Trimethyl borate $(1.15 \mathrm{~mL}, 10 \mathrm{mmol})$ was added in one portion at $-78{ }^{\circ} \mathrm{C}$. After the mixture was warmed to room temperature and following the general workup procedure, the desired product was isolated as a white solid $(0.7$ g, 62\%): mp 96-97 ${ }^{\circ} \mathrm{C}$ (lit. $\left.{ }^{17} \mathrm{mp} 97-99{ }^{\circ} \mathrm{C}\right) ;{ }^{1} \mathrm{H} \mathrm{NMR}\left(\mathrm{CDCl}_{3}\right.$ $400 \mathrm{MHz}) \delta 4.37(\mathrm{~s}, 4 \mathrm{H}), 7.54(\mathrm{~d}, \mathrm{~J}=8.31 \mathrm{~Hz}, 2 \mathrm{H}), 7.67(\mathrm{~d}, \mathrm{~J}=$ $8.31 \mathrm{~Hz}, 2 \mathrm{H}) ;{ }^{13} \mathrm{C} \mathrm{NMR}\left(\mathrm{CDCl}_{3}, 100 \mathrm{MHz}\right) \delta 66.1,126.5,131.1$, 136.3; MS (EI, m/z) 225 (100); HRMS (EI) calcd for $\mathrm{C}_{8} \mathrm{H}_{8} \mathrm{~B}$ ${ }^{79} \mathrm{BrO}_{2}$ 225.9801, found 225.9799; Calcd for $\mathrm{C}_{8} \mathrm{H}_{8} \mathrm{~B}^{81} \mathrm{BrO}_{2} 227.9780$, found 227.9784 .

Acknowledgment. We thank the National Science Council and Ministry of Education of Taiwan for the financial support. Wealso thank Miss Sun Shu-Yun for the HRMS analysis.

Supporting Information Available: ${ }^{1} \mathrm{H}$ and ${ }^{13} \mathrm{C}$ NMR spectra (PDF) for all arylboronic esters. This material is available free of charge via the Internet at http://pubs.acs.org.

J 0011073] 